\title{
Biosynthesis and molecular regulation of secondary metabolites in microorganisms
}

\author{
Yue $\mathrm{Li}^{1} \&$ Huarong $\operatorname{Tan}^{1,2 *}$ \\ ${ }^{1}$ Institute of Microbiology, Chinese Academy of Sciences, Beijing 100101, China; \\ ${ }^{2}$ University of Chinese Academy of Sciences, Beijing 100049, China
}

Received May 8, 2017; published online August 1,2017

Citation: $\quad$ Li, Y., and Tan, H. (2017). Biosynthesis and molecular regulation of secondary metabolites in microorganisms. Sci China Life Sci 60, 935-938. doi: $10.1007 / \mathrm{s} 11427-017-9115-\mathrm{x}$

Antibiotics are most important compounds in microbial secondary metabolites. As we know, streptomycetes are a particularly abundant source of antibiotics and related compounds, providing more than half of medically important antimicrobial and antitumor agents. Various environmental and physiological conditions influence the onset and level of antibiotic production. Because of improper use and abuse of antibiotics as well as the horizontal transfer of antibiotic resistance genes between bacteria by conjugation, transduction or transformation, these have led to the appearance of antibiotic resistance and the loss of antibiotic native efficiency. Therefore, it is necessary to find new antibiotics and to study their clinical application. Here we present a comprehensive review including strategies for discovery of novel antibiotics, novel mechanism of biosynthesis and regulation of antibiotics, and improvement of industrial antibiotics-producers. In this issue, eight articles contributed by Linquan Bai, Yihua Chen, Keqiang Fan, Gang Liu, Yinhua Lu and Huarong Tan primarily concern biosynthesis and molecular regulation of important antibiotics.

\section{DISCOVERY OF NOVEL ANTIBIOTICS}

Sequencing of the tens of Streptomyces genomes revealed the presence of a large number of secondary metabolic gene clus-

*Corresponding author (email: tanhr@im.ac.cn) ters for previously unsuspected products (Bentley et al., 2002) and thus the potential to produce many more natural products than had previously been recognized. It has therefore become necessary to establish new methods to activate these "cryptic" pathways. Currently used or proposed methods include optimization of culture and fermentation conditions, genetic manipulations of genes or gene clusters, and activation of cryptic secondary metabolite gene clusters mediated by specialized metabolites (Liu et al., 2013; Niu et al., 2016). Possible methods or strategies are proposed as follows: (i) Optimization of culture and fermentation conditions: specific environmental signals or nutritional components required for the activation of cryptic gene clusters may in some cases reflect the presence of interacting microorganisms in the natural environment. Co-culture can be an effective method for activating the production of cryptic metabolites. For example, the co-culture of a Streptomyces with mycolic acid-containing Tsukamurella pulmonis from soil samples can induce the Streptomyces to produce a novel antibiotic (Onaka et al., 2011). It is also noteworthy that Streptomyces rapamycinicus can specifically induce expression of the silent biosynthetic gene clusters in Aspergillus nidulans to result in the production of the archetypal polyketide orsellinic acid (Schroeckh et al., 2009). The activation is achieved by Streptomyces-triggered fungal histone acetylation modifications (Nutzmann et al., 2011). The growing understanding of cross talk between Streptomyces and other species may 
provide more opportunities for the discovery of novel natural products, especially antibiotics. However, even though this approach is effective, it is still laborious and obscure. Therefore, miniaturized high-throughput screening methods need to be used. Traditionally, modifying fermentation conditions have been crucial for industrial yield improvement. Some of the molecular information described in this review may help to make such exploration, and permit greater interplay between fermentation development and targeted genetic manipulations. However, empirical observations may continue to be useful, for example, it was recently reported that addition of the rare earth scandium to the fermentation medium significantly stimulated the production of actinorhodin in $S$. coelicolor, actinomycin in S. antibioticus, and streptomycin in S. griseus (Kawai et al., 2007). Genomic techniques may also be useful: based on the transcriptome analysis, several novel compounds were isolated from $S$. flaveolus by using six different media (Qu et al., 2011). These successful results suggest that combining changes of fermentation conditions with -omic studies may even identify ways of awakening cryptic gene clusters for novel compounds. (ii) Genetic manipulations of genes or gene clusters: it is possible to activate the expression of cryptic gene cluster by genetic manipulation of CSR genes encoding activators or repressors (Liu et al., 2013). A straight forward strategy, simply to overexpress activators or delete repressors, has proved effective. For example, overexpression of polR resulted in two-fold increase of polyoxin in S. cacaoi. Moreover, a giant type I modular PKS gene cluster of $S$. ambofaciens ATCC23877, spanning almost $150 \mathrm{~kb}$, was activated by the overexpression of a LAL family regulatory gene, leading to the discovery of stambomycins, unusual glycosylated macrolides with unique chemical structures and promising antiproliferative activity against human cancer cell lines (Laureti et al., 2011). Similarly, the activation of the cryptic cpk gene cluster and production of a novel antibiotic followed the deletion of $s c b R 2$ in $S$. coelicolor, and likewise deletion of the $s c b R 2$ homologue jadR2 in $S$. venezuelae activated jadomycin B production in the absence of the normally required stress challenges (Xu et al., 2010). The inactivation of a putative repressor gene pgaY in Streptomyces sp. PGA64 resulted in the production of two major angucycline metabolites, which had not been detected in the parental wild-type strain (Liu et al., 2013; Metsä-Ketelä et al., 2004). For activating cryptic gene clusters, heterologous expression of the gene cluster in different host strains can be used to confirm the integrity of gene clusters synthesizing secondary metabolites (Liu et al., 2013) and for combinatorial biosynthesis to produce novel derivatives of bioactive secondary metabolites. S. coelicolor and S. avermitilis hosts have proved useful in the expression of heterologous gene clusters. (iii) Activation of cryptic gene clusters mediated by specialized metabolites: specialized metabolites drive at specific sig- nalling molecules, such as $\gamma$-butyrolactones (GBL). Lack of these small molecules may be a factor causing the silence of gene clusters in various actinomycetes. The discovery of more signalling molecules and their use will speed up the process of cryptic gene cluster activations and deepen our understanding of the roles of signalling molecules in antibiotic biosynthesis. Hormone-like signalling molecules, also known as diffusible signalling molecules or autoregulators, can elicit antibiotic production and induce morphological differentiation at nanomolar concentrations (Niu et al., 2016). After about half a century, a total of 29 autoregulators to date have been identified from twelve Streptomyces species. It is possible that further classes of autoregulatory signalling molecules regulate the biosynthesis of some antibiotics, but their further discovery is impeded by the fact that they are normally produced in very small quantities. Recent studies have still relied on the traditional large-scale fermentation method for identification of autoregulators (Zou et al., 2014). Therefore, it is necessary to devise rapid methods and strategies to identify much more signalling molecules, and to study their function in antibiotic biosynthesis. In addition, interaction of signalling molecules between Gram positive and Gram negative bacteria is worth studying in inducing antibiotic production.

\section{NOVEL MECHANISM OF BIOSYNTHESIS AND REGULATION OF ANTIBIOTICS}

Synthetic biology can generate unnatural compounds with predictable features (Chao et al., 2015). The successful application of this technology requires a diverse library of biological modules. These modules can be integrated into assemble complex pathways in a programmed fashion. The assembled pathway needs a suitable surrogate host for production, and Escherichia coli is the most commonly used. Alternative hosts, like $S$. coelicolor M1146, have also been successful. By using the well-developed hosts as a cellular factory, we can produce new antibiotics through assembly of designed and compatible modules. Due to the suitability of different biological modules, the synthetic biological strategies for generating novel compounds still need making more efforts. The new hybrid antibiotics have been generated by using combinatorial biosynthesis. Combinatorial biosynthetic approach can produce valuable and novel hybrid antibiotics with improved properties. For example, hybrid antibiotics with the nikkomycin nucleoside and polyoxin peptidyl moieties were obtained (Li et al., 2011). Genes required for the biosynthesis of the dipeptidyl moiety of polyoxin from $S$. cacaoi were introduced into a $S$. ansochromogenes mutant producing the nucleoside moiety of nikkomycin X. Two hybrid antibiotics were generated. One of them was identified as polyoxin A, a novel compound. The hybrid antibiotic exhibited merits from both parents: it had better inhibitory 
activity against phytopathogenic fungi than polyoxin $\mathrm{B}$, and was more stable under different $\mathrm{pH}$ and temperature conditions than nikkomycin X. Regulation of antibiotic biosynthesis is a complex and hierarchy process, it involves the pathway-specific, pleiotropic, cascade, feed-forward and feedback, and cross talk regulations. Some specialized metabolites play an important role in this process, for example, hormone-like signalling molecules (auto-regulators), antibiotics including its precursors and intermediates as signals in interspecies communication. In feed-forward regulation, JadR* is a good example in jadomycin biosynthesis of $S$. venezuelae. JadR* negatively regulates jadomycin biosynthesis mediated by its biosynthetic intermediates 2,3-dehydro-UWM6 (DHU), dehydrorabelomycin (DHR) and jadomycin A (JdA) (Zhang et al., 2013). Meanwhile, RNA regulation is a developing research field in antibiotic production. The depletion of RNase III resulted in increased levels of many transcripts. An alternative explanation is that RNase III is responsible for rRNA maturation, which is important for the translation of long mRNA (Liu et al., 2013). Moreover, it is a remarkable fact that some regulators cannot directly regulate their target genes due to the lack of their DNA binding domain. These proteins have to be associated with others to exert their regulatory function in antibiotic biosynthesis. For example, WhiB, a founding member of the Wbl family, does not act independently but rather coregulates its regulon of sporulation genes with a partner transcription factor, WhiA (Bush et al., 2016)

\section{IMPROVEMENT OF INDUSTRIAL ANTIBIOTICS-PRODUCING STRAINS}

Microorganisms can produce a variety of secondary metabolites, of which the antibiotics are most important. About $60 \%$ of the natural antibiotics are produced by Streptomyces. Improvement of the productivity of commercially antibiotic-producing strains is an important research field in microbiology. Generation of the high-producing strains is crucial for antibiotic production on a large scale. Traditionally, the improvement of antibiotic-producing strain suitable for industrial fermentations is achieved by random mutagenesis and selection techniques. Although these techniques have succeeded in generating many industrial strains, it exhausted time and human resources. Therefore, with the quick development of DNA sequencing, genetic manipulation of genes or gene cluster is expected to improve antibiotic production. For example, overexpression of positive regulatory genes or structural genes encoding specific enzymes in connection with metabolic bottlenecks has significantly overproduced substantial natural products. Meanwhile, improvement of antibiotic productivity is related to its precursors. For example, the gougerotin yield in Streptomyces graminearus was enhanced in the presence of glycine as a precursor, and it was approximately 2.5 -fold higher than the absence of glycine (Jiang et al., 2013). The strategies could be extended to other Streptomyces for improving production. In addition, biosynthetic pathway from the perspective of metabolic flux can change metabolic processes via genetic manipulation. In this case, we can obtain desired products for its valid application or remove undesired compounds for its invalid application.

Compliance and ethics The author(s) declare that they have no conflict of interest.

Acknowledgements This work was supported by grants from the Ministry of Science and Technology of China (2013CB734001) and the National Natural Science Foundation of China (31470206 and 31571281).

Bentley, S.D., Chater, K.F., Cerdeño-Tárraga, A.M., Challis, G.L., Thomson, N.R., James, K.D., Harris, D.E., Quail, M.A., Kieser, H., Harper, D., Bateman, A., Brown, S., Chandra, G., Chen, C.W., Collins, M., Cronin, A., Fraser, A., Goble, A., Hidalgo, J., Hornsby, T., Howarth, S., Huang, C.H., Kieser, T., Larke, L., Murphy, L., Oliver, K., O'Neil, S., Rabbinowitsch, E., Rajandream, M.A., Rutherford, K., Rutter, S., Seeger, K., Saunders, D., Sharp, S., Squares, R., Squares, S., Taylor, K., Warren, T., Wietzorrek, A., Woodward, J., Barrell, B.G., Parkhill, J., and Hopwood, D.A. (2002). Complete genome sequence of the model actinomycete Streptomyces coelicolor A3(2). Nature 417, 141-147.

Bush, M.J., Chandra, G., Bibb, M.J., Findlay, K.C., and Buttner, M.J. (2016). Genome-wide chromatin immunoprecipitation sequencing analysis shows that WhiB is a transcription factor that cocontrols its regulon with WhiA to initiate developmental cell division in Streptomyces. mBio 7, e00523-00516.

Chao, R., Yuan, Y.B., and Zhao, H.M. (2015). Building biological foundries for next-generation synthetic biology. Sci China Life Sci 58, 658-665.

Jiang, L., Wei, J., Li, L., Niu, G., and Tan, H. (2013). Combined gene cluster engineering and precursor feeding to improve gougerotin production in Streptomyces graminearus. Appl Microbiol Biotechnol 97, 10469-10477.

Kawai, K., Wang, G., Okamoto, S., and Ochi, K. (2007). The rare earth, scandium, causes antibiotic overproduction inStreptomyces spp.. FEMS Microbiol Lett 274, 311-315.

Laureti, L., Song, L., Huang, S., Corre, C., Leblond, P., Challis, G.L., and Aigle, B. (2011). Identification of a bioactive 51-membered macrolide complex by activation of a silent polyketide synthase in Streptomyces ambofaciens. Proc Natl Acad Sci USA 108, 6258-6263.

Li, J., Li, L., Tian, Y., Niu, G., and Tan, H. (2011). Hybrid antibiotics with the nikkomycin nucleoside and polyoxin peptidyl moieties. Metab Eng 13, 336-344.

Liu, G., Chater, K.F., Chandra, G., Niu, G., and Tan, H. (2013). Molecular regulation of antibiotic biosynthesis in Streptomyces. Microb Mol Biol Rev 77, 112-143.

Metsä-Ketelä, M., Ylihonko, K., and Mäntsälä, P. (2004). Partial activation of a silent angucycline-type gene cluster from a rubromycin beta producing Streptomyces sp. PGA64. J Antibiot 57, 502-510.

Niu, G., Chater, K.F., Tian, Y., Zhang, J., and Tan, H. (2016). Specialised metabolites regulating antibiotic biosynthesis inStreptomyces spp.. FEMS Microbiol Rev 40, 554-573.

Nutzmann, H.W., Reyes-Dominguez, Y., Scherlach, K., Schroeckh, V., Horn, F., Gacek, A., Schumann, J., Hertweck, C., Strauss, J., and Brakhage, A.A. (2011). Bacteria-induced natural product formation in the fungus Aspergillus nidulans requires Saga/Ada-mediated histone acetylation. Proc Natl Acad Sci USA 108, 14282-14287. 
Onaka, H., Mori, Y., Igarashi, Y., and Furumai, T. (2011). Mycolic acidcontaining bacteria induce natural-product biosynthesis in Streptomyces Species. Appl Environ Microbiol 77, 400-406.

Qu, X., Lei, C., and Liu, W. (2011). Transcriptome mining of active biosynthetic pathways and their associated products in Streptomyces flaveolus. Angew Chem Int Ed 50, 9651-9654.

Schroeckh, V., Scherlach, K., Nützmann, H.W., Shelest, E., Schmidt-Heck, W., Schuemann, J., Martin, K., Hertweck, C., and Brakhage, A.A. (2009). Intimate bacterial-fungal interaction triggers biosynthesis of archetypal polyketides in Aspergillus nidulans. Proc Natl Acad Sci USA
106, 14558-14563.

Xu, G., Wang, J., Wang, L., Tian, X., Yang, H., Fan, K., Yang, K., and Tan, H. (2010). "Pseudo" $\gamma$-butyrolactone receptors respond to antibiotic signals to coordinate antibiotic biosynthesis. J Biol Chem 285, 27440-27448.

Zhang, Y., Pan, G., Zou, Z., Fan, K., Yang, K., and Tan, H. (2013). $\mathrm{JadR}^{*}$-mediated feed-forward regulation of cofactor supply in jadomycin biosynthesis. Mol Microbiol 90, 884-897.

Zou, Z., Du, D., Zhang, Y., Zhang, J., Niu, G., and Tan, H. (2014). A $\gamma$-butyrolactone-sensing activator/repressor, JadR3, controls a regulatory mini-network for jadomycin biosynthesis. Mol Microbiol 94, 490-505.

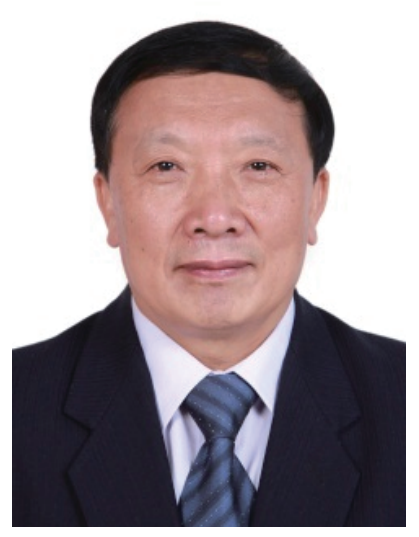

\section{Guest Editor's Biographical Sketch}

Huarong Tan obtained his Ph.D. in microbial genetics in 1991 at the University of East Anglia, England. In 1992, he started to work on the molecular regulation of differentiation and secondary metabolites in Streptomyces. In 1994, he was appointed as full Professor in molecular genetics at the Institute of Microbiology, Chinese Academy of Sciences (CAS). From 1999 to 2008, he served as the Deputy Director of the Institute of Microbiology, CAS. From 2001 to 2011, he was a Vice President of Chinese Society for Microbiology. From 2013 to the present, he has been a Vice President of Chinese Society for Genetics. From 2014 to the present, he has been a member of editorial board for Molecular Microbiology. From 2010 to the present, he has been a member of editorial board for Science China Life Sciences. From 2006 to the present, he has been an Editor in Chief for Acta Microbiologica Sinica. He has published more than 130 papers on his research field. 